\title{
Integration of Motion Signals for Smooth Pursuit Eye Movements
}

\author{
RICHARD T. BORN AND CHRISTOPHER C. PACK \\ Department of Neurobiology, Harvard Medical School, Boston, \\ Massachusetts 02115, USA
}

KEYWORDS: aperture problem; smooth pursuit; motion processing; area MT

How does the brain combine local motion measurements to form an accurate description of object motion? For example, if a vertically oriented bar moves upward and rightward at a constant velocity, a neuron with a small receptive field positioned along the length of the contour can measure only the rightward component of motion, since the upward component provides no time-varying information within its receptive field. In contrast, cells positioned at the endpoints of the contour can measure motion direction accurately. Because direction-selective neurons early in the visual pathways have small receptive fields, the visual system is constantly faced with this "aperture problem." And since they provide the sole input to subsequent stages of cortical visual processing, which in turn inform the premotor circuitry used for making eye movements, the error in measuring local velocity could be perpetuated. How are these conflicting motion signals - the potentially erroneous signals measured along a contour and the correct signals originating from terminators-ultimately resolved in the visual cortex?

Microelectrode recordings from neurons in the middle temporal visual area (MT) of alert monkeys have shown that the earliest directional responses, beginning about $80 \mathrm{msec}$ after the onset of stimulus motion, primarily encode the component of motion perpendicular to the orientation of a contour. That is, they are strongly affected by the ambiguous contour signals. However, the later responses ( $>140 \mathrm{msec}$ after motion onset) encode the true direction of motion, irrespective of contour orientation. Thus the responses of MT neurons reflect the solution of the aperture problem for motion over a period of about $60 \mathrm{msec}^{2}$

Given the evidence that MT neuronal signals are important for the initiation of smooth pursuit eye movements, ${ }^{3-5}$ we asked whether the time-evolving signals we observed in MT neurons had a behavioral correlate. We had subjects (monkeys and humans) track the center of a single moving bar whose orientation was varied with respect to its direction of motion. The bar was $\operatorname{dim}$ green $\left(4.2 \mathrm{~cd} \cdot \mathrm{m}^{-2} ; u^{\prime}=0.28\right.$, $\left.v^{\prime}=0.59\right)$, and its center was indicated by an isoluminant, red gaussian blob

Address for correspondence: Richard T. Born, M.D., Department of Neurobiology, Harvard Medical School, 220 Longwood Ave., Boston, MA 02115-5701. Voice: 617-432-1307; fax: 617734-7557.

rborn@hms.harvard.edu

Ann. N.Y. Acad. Sci. 956: 453-455 (2002). ( 2002 New York Academy of Sciences. 
$\left(4.2 \mathrm{~cd} \cdot \mathrm{m}^{-2} ; u^{\prime}=0.64, v^{\prime}=0.34\right)$. The subject had to track the blob to within $\pm 3^{\circ}$ while eye movements were monitored using a scleral search coil. Eye position and velocity (analog differentiator: low pass, $-3 \mathrm{~dB}$ at $50 \mathrm{~Hz}$ ) were digitized at $1 \mathrm{kHz}$ and stored to disk for off-line analysis.

In agreement with previous studies of both human perception ${ }^{6}$ and the human ocular following response, ${ }^{7}$ the initial direction of pursuit deviated in a direction perpendicular to the orientation of the bar, regardless of the true direction of bar motion. We then asked how the relative amounts of contour and terminator information affected the behavior by varying the length of the bar on randomly interleaved trials. Increasing bar length had the expected effect of increasing the magnitude of the deviation, as measured by the initial eye acceleration perpendicular to the direction of target motion. This effect of bar length was decreased if the targets were presented eccentrically, consistent with the predicted effects of larger receptive field sizes with increased eccentricity (magnification factor).

To study such scaling effects more thoroughly, we next used long bars of varying widths, which had the effect of symmetrically displacing the ambiguous contour signals various distances from the fovea. The "bars" for these experiments were actually parallelograms (i.e., end contour parallel to direction of bar motion), chosen so that we did not introduce potentially disambiguating contour signals at the ends of the bar. These results showed the same effect described above (i.e., smaller deviations with greater eccentricities) and also afforded us the opportunity to examine longer periods of pursuit uninterrupted by saccades. Interestingly, the earliest period (0 to $40 \mathrm{msec}$ from the onset of pursuit) showed a relatively shallow, nearly linear decrement in perpendicular eye acceleration as a function of eccentricity, whereas the later period (40 to $80 \mathrm{msec}$ ) showed a much steeper, exponential decline. This differential sensitivity to eccentricity is reminiscent of a similar difference found for initial eye acceleration as a function of target position ${ }^{8}$ and supports the idea that the early phase of pursuit initiation is driven by inputs that de-emphasize the central visual fields, ${ }^{9}$ or, alternatively, have uniformly large receptive fields across the representation of visual space — such as those from the accessory optic system. ${ }^{10}$ In this scheme, the later phase of pursuit initiation would be driven by the cortical pathway involving area MT, which inherits a considerable emphasis on foveal vision from the striate cortex and thus exhibits an exponential decline in magnification factor. ${ }^{11}$

Finally, given the robust nature of the "bottom-up" contour effects on pursuit, we thought it would be interesting to pit these signals against more "top-down" signals based on the predictability of target motion. We performed two types of experiments in both monkeys and humans. In the first, target motion on any given trial could be in one of four possible directions, but, on half of the trials, target onset was preceded by a cue that indicated the direction of motion of the upcoming target. In the second type of experiment, the direction of motion was exactly the same on every trial. For the monkeys, neither one of these manipulations affected the magnitude or timing of the contour-induced deviation; however, we as yet have no independent evidence that the monkey made use of the predictive signals. For human observers, predictability reduced the effect by about $50 \%$.

In sum, our results indicate that the primate pursuit system initially derives a rough estimate of direction by averaging local motion signals obtained from neurons with relatively small receptive fields. The same effect of ambiguous contour motion 
has been observed in human perception, ${ }^{6}$ the human ocular following response, ${ }^{7}$ and the responses of MT neurons. ${ }^{2}$ The initial integration takes place over a remarkably large spatial range - up to $35^{\circ}$ - further blurring the distinction between smooth pursuit and other smooth eye movements, such as ocular following and the optokinetic response. The efficacy of spatial integration depends on both eccentricity and the period of pursuit measured, perhaps constituting a signature of early, subcortical influences followed by later, cortical signals. Lastly, in humans top-down signals, such as prior knowledge of true target direction, can clearly reduce the earliest contourinduced effect, but they cannot eliminate it completely.

\section{REFERENCES}

1. Marr, D. \& S. Ullman. 1981. Directional selectivity and its use in early visual processing. Proc. R. Soc. Lond. B Biol. Sci. 211: 151-180.

2. PACK, C.C. \& R.T. Born. 2001. Temporal dynamics of a neural solution to the aperture problem in visual area MT of macaque brain. Nature 409: 1040-1042.

3. Newsome, W.T., R.H. Wurtz, M.R. Dursteler \& A. Mikami. 1985. Deficits in visual motion processing following ibotenic acid lesions of the middle temporal visual area of the macaque monkey. J. Neurosci. 5: 825-840.

4. Groh, J.M., R.T. Born \& W.T. Newsome. 1997. How is a sensory map read out? Effects of microstimulation in visual area MT on saccades and smooth pursuit eye movements. J. Neurosci. 17: 4312-4330.

5. Born, R.T., J.M. Groh, R. ZhaO \& S.J. Lukasewycz. 2000. Segregation of object and background motion in visual area MT: effects of microstimulation on eye movements. Neuron 26: 725-734.

6. Lorençeau, J., M. Shiffrar, N. Wells \& E. Castet. 1993. Different motion sensitive units are involved in recovering the direction of moving lines. Vision Res. 33: 12071217.

7. Masson, G.S., Y. Rybarczyk, E. Castet \& D.R. Mestre. 2000. Temporal dynamics of motion integration for the initiation of tracking eye movements at ultra-short latencies. Vis. Neurosci. 17: 753-767.

8. Lisberger, S.G. \& L.E. Westbrook. 1985. Properties of visual inputs that initiate horizontal smooth pursuit eye movements in monkeys. J. Neurosci. 5: 1662-1073.

9. Lisberger, S.G., E.J. Morris \& L. Tychsen. 1987. Visual motion processing and sensory-motor integration for smooth pursuit eye movements. Annu. Rev. Neurosci. 10: $97-129$.

10. Simpson, J.I. 1984. The accessory optic system. Annu. Rev. Neurosci. 7: 13-41.

11. Daniel, P.M. \& D. Whitteridge. 1961. The representation of the visual field on the cerebral cortex in monkeys. J. Physiol. 159: 203-221. 